\title{
FRATURA TRAUMÁTICA DE COLUNA TORÁCICA TI-T10
}

\author{
Asdrubal Falavigna ${ }^{1}$, Orlando Righesso Neto ${ }^{2}$, \\ Fernando Antonio Patriani Ferraz ${ }^{3}$, Márcio Manozzo Boniatti ${ }^{4}$
}

\begin{abstract}
RESUMO - Descrevemosa incidência, causas, manejo e prognóstico dasfraturastraumáticas de coluna torácica de T1 a T10. Estudamos retrospectivamente os casos cirúrgicos de fraturas traumáticas da coluna torácica (T1 a T10), durante o período de junho de 1994 a junho de 2003. 0 tipo de fratura foi determinado segundo a classificação de Gertzbein e o grau de estabilidade através da classificação de Denis. O quadro neurológico à internação e 30 dias após a cirurgia foi avaliado através da classificação de ASIA/IMSOP. O objetivo da cirurgia nos pacientes com lesão medular completa $(n=7)$ foi de estabilização, enquanto nos casos de instabilidade vertebral em que não houve lesão medular ou esta foi incompleta $(n=12)$, optou-se pela descompressão do tecido nervoso, correção do alinhamento da coluna e estabilização da coluna.
\end{abstract}

PALAVRAS-CHAVE: coluna torácica, fratura traumática, diagnóstico, tratamento, prognóstico.

\begin{abstract}
Traumatic fracture of the thoracic spine T1-T10
ABSTRACT - We describe the incidence, causes, management and prognosis of traumatic fractures of the thoracic spine from T1 to T10 in surgical cases of traumatic fractures of spine during the period from J une 1994 to June 2003 studied retrospectively. The type of fracture was determined according to the Gertzbein classification, and the degree of stability using the Denis classification. The neurological picture at admission and 30 days after surgery was evaluated using the ASIA/IMSOP classification. Surgery was performed in patients with complete spinal cord injury $(n=7)$ for the purpose of stabilization using the posterior approach. In caseswithout spinal cord injury or incomplete injury $(n=12)$, the surgical procedure wasperformed aiming to decompress the nerve tissue, to correct the alignment of the spine and to stabilize the spine.
\end{abstract}

KEY WORDS: thoracic spine, traumatic fracture, diagnosis, treatment, prognosis.

Osacidentes são a quarta maior causa de morte nos Estados Unidos, sendo responsáveis por aproximadamente 50 mortes por 100000 habitantes a cada $a n 0^{1}$. As lesões agudas da coluna vertebral e da medula espinhal estão entre as causas mais freqüentes de incapacidade severa e morte após trauma ${ }^{2-5}$.

Nas fraturas de coluna vertebral, a região cervical e a transição toracolombar são os segmentos mais comumente atingidos $s^{6-8}$. A pesar disso, o segmento torácico superior e médio, de Tl a T10, merece atenção por reapresentar incidência aproximada de $17 \%$ dasfraturas da coluna vertebral, possuir biomecânica própria e, conseqüentemente, ser um tipo de fratura maissusceptível e exibir elevada taxa de lesão neurológica, em torno de $53 \%$, em dois terços ocorrendo lesão medular completa ${ }^{7,8}$.
O objetivo deste estudo é descrever a incidência, ascausas, o manejo e o prognóstico dasfraturas traumáticas de coluna torácica de T1 a T10.

\section{MÉTODO}

Foram estudados retrospectivamente os casos cirúrgicos de fraturastraumáticas da coluna torácica (T1 - T10) durante o período de junho de 1994 a junho de 2003.

$O$ diagnóstico da fratura foi feito através de exames de imagem: raios-X simples, tomografia computadorizada e ressonância magnética da coluna vertebral. $O$ tipo de fratura foi determinado segundo a dassificação de Gertzbein $^{9}$ e o grau de estabilidade através da classificação de Denis $s^{10,11}$. O quadro neurológico pré e pós-operatório foi avaliado através da classificação de ASIA/IM SOP12.

A indicação cirúrgica foi devida a instabilidade da coluna vertebral, deformidade severa e/ou compressão medular.

\footnotetext{
1Professor da Disciplina de Neurologia da Universidade de Caxias do Sul, RSBrasil, Pós-graduando em Neurocirurgia pela Universidade Federal de São Paulo - Escola Paulista de Medicina São Paulo SP, Brasil (UNIFESP-EPM); ${ }^{2}$ Professor da Disciplina de Ortopedia da Universidade de Caxias do Sul; ${ }^{3}$ Coordenador da Disciplina de Neurocirurgia do Departamento de Neurologia e Neurocirurgia da (UNIFESP-EPM ); ${ }^{4}$ Acadêmico da Faculdade de Medicina da Universidade de Caxias do Sul.
}

Recebido 31 Março 2004, recebido na forma final 24 Junho 2004. Aceito 11 Agosto 2004.

Dr. Asdrubal Falavigna - Rua Coronel Camisão 241/301 - 95034-000 Caxias do Sul RS - Brasil. E-mail: asdrubal@doctor.com 
Tabela 1. Casos cirúrgicos de trauma raquemedular $(\mathrm{N}=116)$.

\begin{tabular}{lc}
\hline Segmento da coluna & $\begin{array}{c}\text { Número de } \\
\text { pacientes (\%) }\end{array}$ \\
\hline Cervical & $34(29,3 \%)$ \\
Torácico (T1-T10) & $19(16,3 \%)$ \\
Tóraco-lombar (T11-L2) & $47(40,5 \%)$ \\
Lombar (L3-L5) & $13(11,2 \%)$ \\
Sacral & $2(1,7 \%)$ \\
\hline
\end{tabular}

\section{RESULTADOS}

Durante o período de estudo foram tratadoscirurgicamente 116 casos de fratura de coluna vertebral, sendo $19(16,3 \%)$ no segmento torácico de T1 a T10 (Tabela 1). A média de idade foi 31 anos, havendo predomínio do sexo masculino (17 pacientes, $89,5 \%$ ). A causa mais comum do trauma foi acidente de trânsito, que ocorreu em 14 casos (Tabela 2).

Fraturas em outros segmentos da coluna vertebral foram observadasem 3 casos: 1 no segmento cervical alto (C1-C2) e 2 na região lombar. Fraturas

Tabela 2. Casos cirúrgicos de fratura do segmento torácico de T1 a T10.

\begin{tabular}{|c|c|c|c|c|c|c|}
\hline Casos & $\begin{array}{l}\text { Idade } \\
\text { /Sexo }\end{array}$ & Etiologia & $\begin{array}{l}\text { Classificação } \\
\text { da lesão } \\
\text { Tipo: Grupo }\end{array}$ & $\begin{array}{c}\text { Escala de } \\
\text { ASIA/IMSOP } \\
\text { Internação }\end{array}$ & $\begin{array}{c}\text { Escala de } \\
\text { ASIA/IMSOP } \\
30 \text { dias PO }\end{array}$ & Tipo de Cirurgia \\
\hline 1 & 23/M & Acidente motociclístico & Compressão: Burst T4 & $\mathrm{E}$ & $\mathrm{E}$ & Hartshill \\
\hline 2 & $24 / M$ & Acidente automobilístico & $\begin{array}{l}\text { Translação: ântero-posterior } \\
\text { T5-T6 }\end{array}$ & $A$ & $A$ & Harrington \\
\hline 3 & $56 / M$ & Queda & $\begin{array}{l}\text { Compressão: Burst T5 } \\
\text { Impactação T7 }\end{array}$ & $\mathrm{E}$ & $\mathrm{E}$ & Hartshill \\
\hline 4 & 19/M & Acidente automobilístico & $\begin{array}{l}\text { Translação: ântero-posterior } \\
\text { T5-T6 }\end{array}$ & $A$ & $A$ & Hartshill \\
\hline 5 & $33 / \mathrm{M}$ & Acidente automobilístico & Translação: lateral T10-T11 & $A$ & $A$ & Hartshill \\
\hline 6 & $36 / M$ & Queda & Compressão: Burst T4 e T5 & C & $\mathrm{D}$ & Harrington \\
\hline 7 & $25 / F$ & Acidente automobilístico & $\begin{array}{l}\text { Translação: ântero-posterior } \\
\text { T3-T4 }\end{array}$ & $A$ & $A$ & Hartshill \\
\hline 8 & $28 / F$ & Acidente automobilístico & Compressão: Burst T6 & E & E & VA, VP com CD \\
\hline 9 & $57 / M$ & Queda & Compressão: Impactação T5 & E & E & VP com CD \\
\hline 10 & $40 / \mathrm{M}$ & Acidente motociclístico & Compressão: Burst T9 & E & E & VA com Z-plate \\
\hline 11 & 19/M & Acidente automobilístico & Compressão: Burst T6 & $\mathrm{E}$ & $\mathrm{E}$ & VA Synthes \\
\hline 12 & $25 / \mathrm{M}$ & Acidente motociclístico & $\begin{array}{l}\text { Compressão: Impactação T7 } \\
\text { e BurstT10 }\end{array}$ & E & $\mathrm{E}$ & $\begin{array}{l}\text { VA com } \\
\text { Z-plate (2) }\end{array}$ \\
\hline 13 & $20 / M$ & Acidente motociclístico & Translação: lateral T4-T5 & A & $A$ & Hartshill \\
\hline 14 & $43 / \mathrm{M}$ & Queda & Translação: lateral T9-T10 & $A$ & $A$ & Hartshill \\
\hline 15 & $26 / M$ & Acidente motociclístico & Compressão: Burst T5 e T6 & $\mathrm{E}$ & $\mathrm{E}$ & Hartshill \\
\hline 16 & $17 / \mathrm{M}$ & Acidente automobilístico & Compressão: Burst T8 & $\mathrm{E}$ & $\mathrm{E}$ & VP com CD \\
\hline 17 & 29/M & Queda & Translação: lateral T7-T8 & A & $A$ & VP com CD \\
\hline 18 & $45 / M$ & Acidente automobilistico & $\begin{array}{l}\text { Compressão: Impactação } \\
\text { T6 e Burst T11 }\end{array}$ & $\mathrm{E}$ & $\mathrm{E}$ & VP com CD \\
\hline 19 & $24 / M$ & Acidente motociclistico & $\begin{array}{l}\text { Compressão: Impactação } \\
\text { T7, T8, T9 }\end{array}$ & $E$ & $E$ & $\begin{array}{l}\text { VP com CD } \\
\text { Lesão associada } \\
\text { tórax e abdome }\end{array}$ \\
\hline
\end{tabular}

M, masculino; F, feminino; PO, pós-operatório; VA, via anterior; VP, via posterior; CD, sistema de fixação interna posterior Cotrel-Dubousset; Z, plateplaca anterior de fixação interna E, normal; A, lesão completa; C, lesão incompleta; D, melhora motora. 


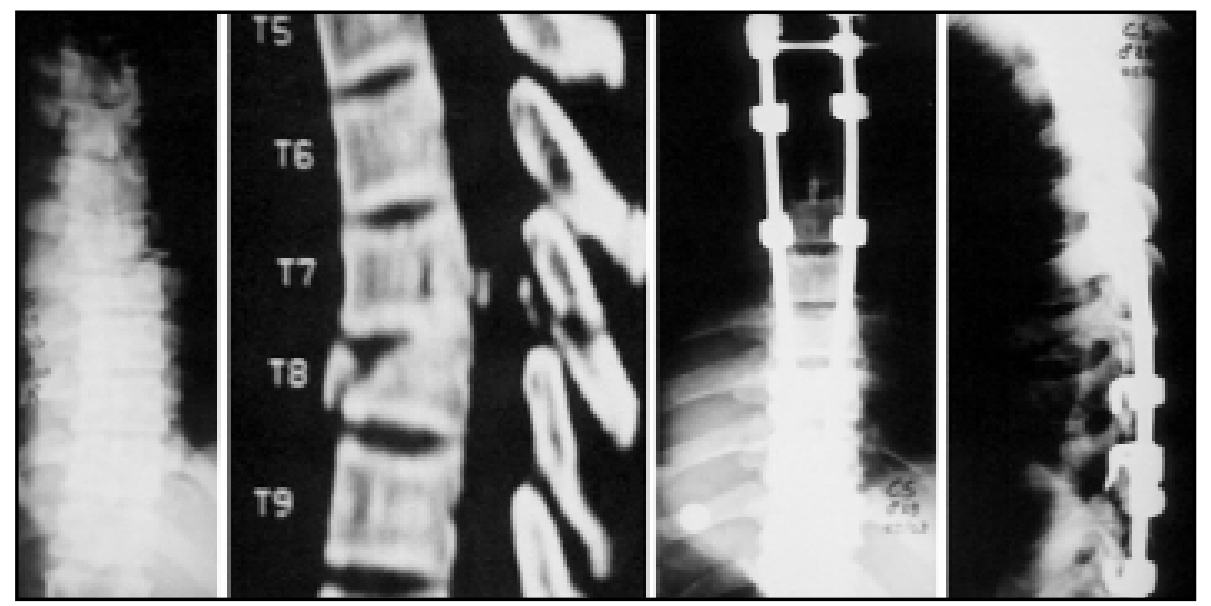

Fig 1. Caso 17. O exame de raios $X$ simples de coluna mostra uma translação lateral T7-T8 que foi corrigida pela via posterior através do uso de CD.

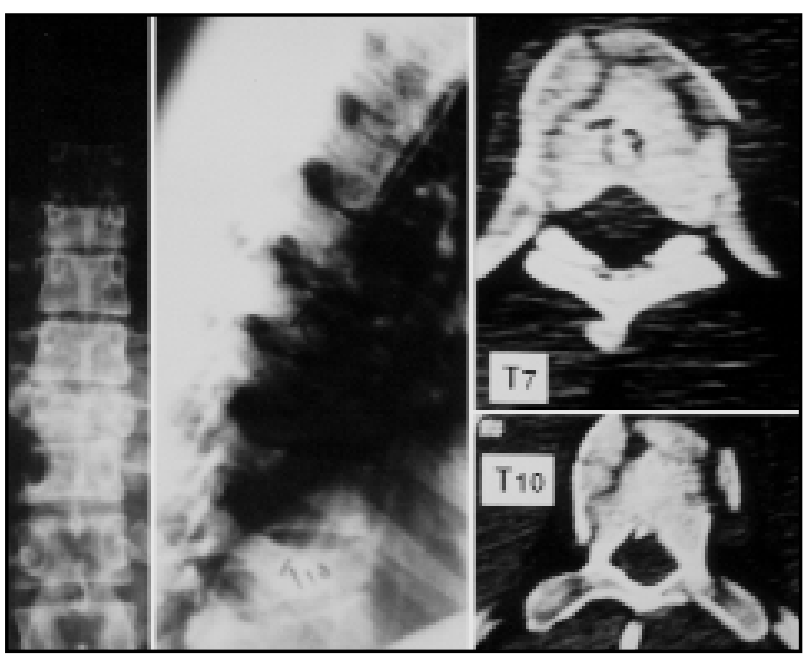

Fig 2. Caso 12. Fratura compressão com impactação de T7 e explosão de 110 com visualização através dos exames de raios$X$ simples, em vista ântero-posterior e lateral, e de tomografia computadorizada em corte axial.

múltiplas do segmento torácico de T1 a T10 foram encontradas em 4 casos, sendo o exame neurológico de admissão hospitalar normal em todos eles. Houve lesão associada de tecido pulmonar em 2 casos e, em 1 caso, de fígado e alça intestinal.

A escala de ASIA/IMSOP quando da internação foi normal (grau E) em 11 casos $(57,8 \%)$, com lesão completa (grau A) em 7 casos (36,8\%) e, em 1 caso (5,3\%), com lesão incompleta (grau C). Não houve modificação do quadro neurológico com a intervenção cirúrgica nos pacientes grausE e A, havendo melhora motora do paciente em grau $C$, que passou a ter capacidade de deambulação com apoio de muletas (Tabela 2).

O objetivo da cirurgia nos pacientes com lesão medular completa $(n=7)$ foi de estabilização, sendo utilizada a via posterior. Nos pacientes sem lesão medular ou com lesão incompleta (12 casos), o procedimento cirúrgico foi realizado como intuito de descomprimir o tecido nervoso, corrigir o alinhamento da coluna e estabilizar a coluna (Figs 1, 2 e 3). $O$ acesso cirúrgico pela via anterior foi utilizado em 3 desses casos, o posterior em 8 e a combinada em 1 (Tabela 2).

\section{DISCUSSÃO}

Asfraturasna coluna torácica são maisfreqüentesno sexo masculino, numa proporção de 4:1, sendo mais comuns em adultos jovens ${ }^{9,13-15}$. Hanley e Eskay $^{8}$ verificaram que a incidência de fratura no segmento torácico de T2 a T12 foi de $16,4 \%^{14,15}$. A causa maiscomum de fraturas de coluna vertebral são os acidentes automobilísticos, responsáveis por aproximadamente 40 a $45 \%$ das lesões, seguida por quedas de altura (20\%), atividades esportivas $(15 \%)$, violência $(15 \%)$ e atividades diversas $(5 \%)^{6}$. Robertson et al. ${ }^{13}$ verificaram que a coluna torácica é a região maisfreqüentemente acometida nosacidentes com motocicletas. Nos casos apresentados de fratura da coluna torácica T1 a T10, a incidência $(16,3 \%)$, a etiologia traumática $(73,7 \%)$ e a preponderância do sexo masculino $(89,5 \%)$ coincidem com os achados da literatura.

A coluna torácica exibe características anatômicasque se relacionam com a biomecânica do trau$\mathrm{ma}^{16}$. A caixa torácica restringe em $70 \%$ o movimento da coluna torácica, especialmente na extensão do tronco, e aumenta quatro vezes sua tolerância à compressão pelo fato das costelas fornecerem força e capacidade de absorção da energia $^{16,17}$. Devido a essesfatores de proteção, é neces- 


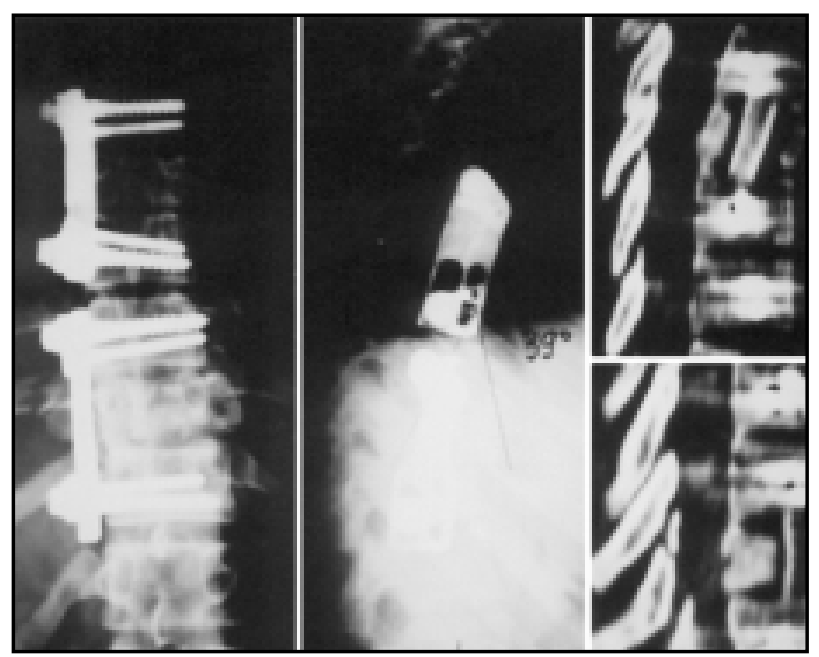

Fig 3. Caso 12. Abordagem anterior com correção da cifose através do uso de enxerto ósseo de ilíaco e de duas placas do tipo Z-plate.

sário um trauma muito mais violento para romper a integridade da coluna torácica do que, por exemplo, a da coluna cervical, o que explica a alta incidência de lesões associadas à fratura de coluna torácica ${ }^{18}$. Somado a isso, existe o fator anatômico que predispõe à lesão medular, pois, no segmento torácico, o diâmetro do canal vertebral é o mais reduzido, a ponto de tais pacientesterem o pior prognóstico para recuperação funcional se comparados com os que sofreram lesão em outros segmentos da coluna vertebral ${ }^{19}$. A incidência de lesão medular foi de 8 casos $(42,1 \%)$; em 7 desses houve lesão neurológica completa.

Várias são as classificações para asfraturas da coluna torácica. Utilizamos a que combina característicasmorfológicas e mecânicas ${ }^{9}$. Tal classificação divide as lesões em tipos A ou compressão, B ou distração e C ou multidirecional com translação. Cada um desses tipos é subdividido em 3 grupos de acordo com asalteraçõesmorfológicase o potencial de instabilidade. Esses grupos, por sua vez, são dicotomizadosem subgrupos conforme o grau de lesão óssea e ligamentar. As fraturas são colocadas em ordem progressiva de severidade da lesão, ou seja, à medida que se passa de uma lesão tipo A para outra de tipo $C$, aumenta a probabilidade de instabilidade mecânica e lesão nervosa. Hanley e Eskay ${ }^{8}$ verificaram que ostipos de fraturas maisfreqüentes no segmento torácico de T2 a T12 foram do tipo deslocamento $(49,1 \%$ ) e compressão $(43,9 \%)$.

A fratura do tipo compressão é a lesão maisfreqüente da coluna torácica ${ }^{15,20,21}$. Este tipo de lesão foi identificado em $52,5 \%$ e $54,2 \%$ dasfraturas ${ }^{10,21}$.
A fratura compressão por explosão é usualmente instável, sendo esponsável por $20 \%$ dasfraturas da coluna torácica e determinando comprometimento nervoso em $47 \%$ dos $\operatorname{casos}^{10,19,21}$. Neste estudo, a fratura por compressão foi detectada em 12 casos (63,1\%). Em discordância com a literatura, a lesão medular foi observada somente em 1 caso. A fratura do tipo distração é usualmente verificada na região toracolombar (T11-L2), estando associada a acidente automobilístico com uso de cinto de segurança baixo. No segmento torácico de T1 a T10 sua incidência é menor, em torno de $2,8 \%{ }^{21}$. A fratura-luxação é caracterizada por lesão em todasas colunas, sendo extremamente instável e com elevada incidência de lesão medular ${ }^{10,19}$. Sua incidência varia de $23 \%$ a $37,5 \%{ }^{10,21}$. No presente estudo, houve 7 casos de fratura-luxação por translação, cuja apresentação neurológica foi, na sua totalidade, de lesão medular completa.

\section{Manejo}

O tratamento das fraturas da coluna torácica permanece controverso. Na literatura existem estudos que recomendam o tratamento cirúrgico, e outros, o não-cirúrgico ${ }^{18,22,23}$. Asfraturas de coluna torácica, em alguns casos, podem ser manejadas com eficácia através do tratamento não-cirúrgico, embora seja necessário maior tempo de repouso e de hospitalização ${ }^{19}$. Petitjean et al. ${ }^{18}$ afirmam que três pontos devem ser considerados para a realização de cirurgia precoce: a existência de compressão medular, o grau do dano neurológico e a presença de lesões associadas. A descompressão cirúrgica precoce no paciente com lesão medular completa não tem indicação, pois a recuperação neurológica é rara, havendo somente a necessidade de estabilização cirúrgica para prevenção de deformidades cifóticas ${ }^{18}$. Para esses pacientes, alguns estudos mostram que o tempo de hospitalização é menor com a estabilização cirúrgica ${ }^{18,23}$. Na presença de compressão medular associada à lesão neurológica parcial, há indicação de descompressão do canal vertebral, alinhamento da coluna vertebral e fixação interna ${ }^{18,24-26}$. Pacientes com fratura de coluna torácica submetidosà cirurgia nas primeiras 72 horas apresentam pós-operatório melhor do que aqueles em que a estabilização é postergada. A fixação precoce resulta em menos tempo de permanência na unidade de tratamento intensivo e no hospital, menor necessidade de suporte ventilatório e redução da taxa de complicações pulmonares. 
A incidência de fratura no segmento torácico de T1 a T10 foi $16,3 \%$, sendo o acidente de trânsito a causa mais comum $(73,7 \%)$. O objetivo da cirurgia nos pacientes com lesão medular completa foi de estabilização e, nos casos sem comprometimento neurológico ou com comprometimento parcial, o de descompressão nervosa, correção do alinhamento e estabilização.

\section{REFERÊNCIAS}

1. Yashon D. Spinal injury. Norwalk: Appleton-Century-Crofts, 1986:86-145.

2. Bohlman $\mathrm{HH}$. Acute fractures and dislocations of the cervical spine: an analysis of 300 hospitalized patients and review of the literature. J Bone Joint Surg 1979;61:1119-1142.

3. Bohlman HH, Ducker TB, Lucas JT. Spine and spinal cord injuries. In Rothman RH, Simeone FA (eds). The spine. Philadelphia: Saunders, 1982:661-757.

4. Bohlman HH. Complications of treatment of fractures and dislocations of the cervical spine. In Epps C (ed). Complications of orthopaedic surgery. Philadelphia: Lippincott, 1985:897-918.

5. Bolesta MJ, Bohlman HH. Late complications of cervical fractures and dislocations and their surgical treatment. In Frymoyer J (ed). The adult spine: principles of practice. New York: Raven Press 1991:1107-1126.

6. Ducker T, Perot P. National spinal cord injury registry. Charleston SC: U.S. Department of Defense, 1975.

7. Fessler RG, Masson RL. Management of thoracic fractures. In Menezes AH, Sonntag VKH (eds). Principles of spinal surgery. New York: McGraw-Hill; 1995:899-918.

8. Hanley EN, Eskay ML. Thoracic spine fractures. Orthopaedics 1989;12:689-696.

9. Gertzbein SD. Spine update: classification of thoracic and lumbar fractures. Spine 1994;19:626-628.

10. Denis $F$. The three column spine and its significance in the classification of acute thoracolumbar spinal injuries. Spine 1983;8:817-831.
11. Denis F. Spinal instability as defined by the three-column spine concept in acute spinal trauma. Clin Orthop 1984;189:65-76.

12. International Standards for Neurological and Functional Classification of Spinal Cord Injury. Revised, 1992. American Spinal Injury Association, International Medical Society of Paraplegia - ASIA/IMSOP 1992.

13. Robertson A, Giannoudis PV, Branfoot T, Barlow I, Matthews SJ, Smith RM. Spinal injuries in motorcycle crashes: patterns and outcomes. J Trauma 2002;53:5-8.

14. Nadalo LA, Moody JA. Thoracic spine, trauma. Emedicine 2002.

15. Oliveira PAS, Pires JV, Borges JMM Filho. Traumatismos da coluna torácica e lombar: avaliação epidemiológica. Rev Bras Ortop 1996;31:771-776.

16. El-Khoury GY, Whitten CG. Trauma to the upper thoracic spine: anatomy, biomechanics, and unique imaging features. Am J Roentgenol 1993;160:95-102.

17. White AA, Panjabi M. Clinical biomechanics of the spine. Philadelphia: Lippincott; 1990:115-186.

18. Petitjean ME, Mousselard H, Pointillart V, Lassie P, Senegas J, Dabadie P. Thoracic spinal trauma and associated injuries: should early spinal decompression be considered? J Trauma 1995;39:368-372.

19. Madsen PW, Lee TT, Eismont FJ, Green BA. Diagnosis and management of thoracic spine fractures. In Youmans JR (ed). Neurological surgery, Vol. 3. Philadelphia: Saunders; 1996:2043-2078.

20. McAfee PC, Yuan HA, Fredrickson BE, Lubicky JP. The value of computed tomography in thoracolumbar fractures: an analysis of one hundred consecutive cases and a new classification. J Bone Joint Surg Am 1983;65:461-473.

21. Argenson C, Boileau P, de Peretti F, Lovet J, Dalzotto H. Fractures of the thoracic spine (T1-T10): a proposal of 105 cases. Rev Chir Orthop Reparatrice Appar Mot 1989;75:370-386.

22. Rechtine GR. Nonsurgical treatment of thoracic and lumbar fractures. Instr Course Lect 1999;48:413-416.

23. Place HM, Donaldson DH, Brown CW, Stringer EA. Stabilization of thoracic spine fractures resulting in complete paraplegia: a long-term retrospective analysis. Spine 1994;19:1726-1730.

24. Gertzbein SD, Court-Brown CM, Marks P, et al. The neurological outcome following surgery for spinal fractures. Spine 1988;13:641-644.

25. Edwards CC, Levine AM. Early rod-sleeve stabilization of the injured thoracic and lumbar spine. Orthop Clin Am 1986;17:121-145.

26. Dunn HK. Anterior stabilization of thoracolumbar injuries. Clin Orthop 1984;189:116-124. 